\title{
DIFFERENT TYPES OF PRE ANALYTICAL ERRORS IN CLINICAL BIOCHEMISTRY LAB OF OSIMANIA GENERAL HOSPITAL AND HOW TO MINIMIZE THEIM.
}

KEY WORDS:

\section{Dr. Bushra Hamed*}

\section{Dr. N. Jaya}

$2^{\text {nd }}$ year MD Biochemistry Postgraduate, Osmania Medical College, Hyderabad, Telangana, India. *Corresponding Author

Professor, Department of Biochemistry, Osmania Medical College, Hyderabad,Telangana, India.

OBJECTIVES: To study different types of pre-analytical errors in clinical biochemistry lab and how to minimize them. METHODOLOGY: An observational study was done at Department of Biochemistry , Osmania General Hospital for a period of 2 months from Aug 2020-Sep 2020.During this phase different types of errors were monitored.

RESULTS: During a period of 2 months , 10000 samples were analyzed and among them 400 were found to be having an error. i.e. $4 \%$. Among them Hemolyzed samples (37.5\%), Lipemic samples(25\%), Misidentification of samples(15\%), Insufficient volume(12.5\%) and Sample mixing (10\%).

CONCLUSION: Proper training to nursing staff and phlebotomist regarding use of vacutainer needles instead of syringes and time of collection of samples reduces the error of hemolysis and lipemia. Use of Barcode labels reduces the error of misidentification. Proper education regarding volume of blood to be collected and use of correct vacutainers reduces the error of insufficient volume and sample mixing.

Background: WHAT ARE THE DIFFERENT TYPES OF PREANALYTICALERRORS?

Clinical laboratory plays an important role in patientcentered approach in delivery of health care services, as physicians and surgeons rely on accurate lab results for proper diagnosis and treatment of diseases.(1),(2),(3). Sample testing is divided into three phases and errors can occur in any phase. But pre- analytical phase is more prone for errors and it contributes to majority of total lab errors.(4). Hence it is of great need to identify these errors and take measures to minimize them, as these errors can lead to misdiagnosis and misinterpretation of lab results . Ultimately it will lead to improper treatment and serious threat towards patient's health.(5).

\section{MATERIALS AND METHODS:}

An observational study was done at Department of Biochemistry, Osmania General Hospital , Hyderabad, Telangana for a period of 2 months from Aug 2020-Sep 2020. During this period samples of varied tests received in lab were observed and errors present were noted. Among the received samples i.e. 10000,400 were found to have an error. So error is $4 \%$.

\section{RESULTS:}

\begin{tabular}{|l|l|}
\hline SAMPLES NOTED & ERRORS \\
\hline 1. HEMOLYZED & $37.5 \%$ \\
\hline 2. LIPEMIC & $25 \%$ \\
\hline 3. MISIDENTIFICATION OF SAMPLES & $15 \%$ \\
\hline 4. INSUFFICIENT VOLUME & $12.5 \%$ \\
\hline 5. SAMPLE MIXING & $10 \%$ \\
\hline TOTAL SAMPLES & $100 \%$ \\
\hline
\end{tabular}

Statistical Analysis: By Bar Chart

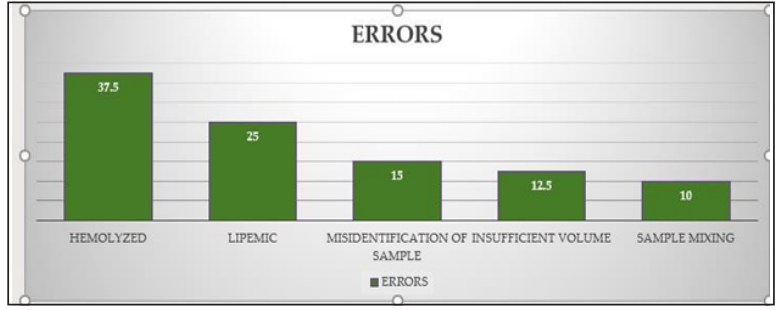

DISCUSSION:

In my study hemolyzed samples accounted for majority of errors i.e. $37.5 \%$. Hemolysis of samples occurs when blood is forced through a fine needle, shaking tubes vigorously.(6). Red colour vacutainers should not be shaken.
Plasma tubes should be gently inverted and repeated freezing and thawing of samples should be avoided as it leads to massive hemolysis.(7).Hemolysis in heel stick blood samples has been attributed in part to mechanical trauma and excessive squeezing at the puncture site. (8).Lipemia is the second common error i.e. $25 \%$. It is due to collection of blood samples after a heavy meal. Misidentification accounting for $15 \%$ is due to improperly filled requisition slips and requisition slips with no identity. Insufficient volume accounting for $12.5 \%$ is due to not filling the vacutainer to the required level, as the blood sample collected for analysis has to be divided among pathology, biochemistry and microbiology departments. Every analytical process requires a fixed volume of serum/ plasma for analysis. It is due to high patient load in government setups.

Sample mixing accounting for $10 \%$ is due to insufficient knowledge regarding correct vacutainers usage and pouring it from one tube to other. Last but not the least all the errors can be attributed to improper knowledge regarding sample collection and analysis.

\section{CONCLUSION:}

Proper training to nursing staff and phlebotomist regarding collection of blood samples in correct vacutainers .

Use of Standard Operating Procedures (SOP) near their working tables will help them to follow step by step instructions for collection of blood samples. By this we can achieve efficiency, quality output and uniformity of performance, while reducing miscommunication and failure. Proper education regardinghemolyzed and lipemic samples like collection of blood for lipid profile in fasting state can reduce lipemic sample. Collection of blood glucose sample after 8-10 hrs of fasting for a fasting sample and for post lunch $2 \mathrm{hrs}$ after meal and while cleaning the skin before collection of blood sample, care should be taken that no alcohol remains on skin as alcohol traces may cause hemolysis. Use of Bar code labelling can reduce misidentification of samples. Best thing is to maintain a data of samples rejected due to various causes and to prevent them in future.

\section{REFERENCES}

1. Datta P. Resolving discordant specimens. ADVANCE for Administrators of the laboratory. July 2005:60.

2. Hawkins R. Managing the pre and post analytical phases of the total testing process. Ann Lab Med.2012;32(1):5-16.

3. Plebani M Errors in clinical laboratories or errors in laboratory medicine. Clin Chem Lab Med 2006;44(6):750-759.

4. Jiu $\mathrm{T}$, The importance of pre analytical phase in lab testing and diagnosis. Eur Clin Chem;Sept 2010. 
5. Green SF . The cost of poor blood specimen quality and errors in pre analytical processes. Clin Biochem .2013;46(13): 1175-1179.

6. Carraro P. Hemolyzed specimens: $A$ reason for rejection or a clinical challenge?Clin Chem .2000;46:306-307.

7. Jay DW Provasek D. Characterization and mathematical correction of hemolysis interference in selected Hitachi 717 assays. Clin Chem .1993;39: 1804-1810.

8. Bhutani VKGourley GRAdler SKreamer BDalin CJohnson LH Noninvasive measurement of total serum bilirubin in a multiracial predischarge newborn population to assess the risk of severe hyperbilirubinemia. Pediatrics. Available athttp://www.pediatrics.org/cgi/content/full/106/2/el7Access ed February 16,2002Google Scholar. 